\title{
Les Déterminants De L'accès Aux Services De Santé À Grand Bassam
}

\author{
Maï Gilles-Harold Wilfried \\ Doctorant, Université Félix Houphouët Boigny \\ Aloko-N'guessan Jérôme \\ Directeur de Recherches, Université Félix Houphouët-Boigny \\ Essan Kodia Valentin \\ Maître Assistant, Institut, Université Félix Houphouët Boigny
}

Doi: 10.19044/esj.2018.v14n6p124 URL:http://dx.doi.org/10.19044/esj.2018.v14n6p124

\begin{abstract}
State of complete physical, mental and social well-being, health is fundamental for man. Yet global figures for access to health are alarming. According to Gijs (2011) 2.5 billion people in the world do not have access to basic health care. In Côte d'Ivoire, the State has made population access a priority. Thus, sanitary infrastructures were built and equipped (PNDS, 2016). However, the problem of access to health services remains. The city of GrandBassam, located in the south-east of Côte d'Ivoire, has a wide range of health structures. Yet there are still people who still do not have access to health care. According to the RASS (2015), 25\% of the population of Grand-Bassam still does not use health services. The purpose of this article is to identify the determinants of the access of the population of Grand-Bassam to health services. The methodological approach adopted to conduct this study took into account a bibliographic synthesis and the administration of a questionnaire. This study shows that perception of distance, income of head of household, perception of cost of care, household size, educational level, and age of households are the factors that determine access health services in the city of Grand-Bassam.
\end{abstract}

Keywords: Access, sanitary infrastructure, Grand-Bassam, Ivory Coast

Résumé

État de bien-être complet physique, mental et social, la santé est fondamentale pour l'homme. Pourtant les chiffres mondiaux en matière d'accès à la santé sont alarmants. Selon Gijs (2011) 2,5milliards de personnes dans le monde n'ont pas accès aux soins de santé de base. En Côte d'Ivoire, l'État a fait de l'accès des populations une priorité. C'est ainsi que des 
infrastructures sanitaires ont été construites et équipées (PNDS, 2016). Cependant le problème de l'accès aux services de santé demeure. La ville de Grand-Bassam, située au sud-est de la Côte d'Ivoire, dispose d'une gamme variée de structures sanitaires. Pourtant l'on trouve encore des habitants qui n'ont toujours pas accès aux soins de santé. Selon le RASS (2015), 25\% de la population de Grand-Bassam n'utilisent toujours pas les services de santé. L'objectif de cet article est d'identifier les déterminants de l'accès des populations de Grand-Bassam aux services de santé. La démarche méthodologique adoptée pour mener cette étude a pris en compte une synthèse bibliographique et l'administration d'un questionnaire. Il ressort de cette étude que la perception de la distance, le revenu du chef de ménage, la perception du coût des soins, la taille des ménages, le niveau d'instruction et l'âge des ménages sont les facteurs qui déterminent l'accès aux services de santé dans la ville de Grand-Bassam.

Mots-clés : Accès, santé, infrastructures sanitaires, Grand-Bassam, Côte d'Ivoire

\section{Introduction}

La question de l'accès aux services de santé est depuis quelques années, au centre des préoccupations des scientifiques et des politiques. La santé est la base du développement intellectuel, physique, affectif et spirituel (OMS, 2013). Le lien entre la santé et la stabilité sociale est tel que la détérioration de l'une entrave le progrès de l'autre et se répercute sur le développement économique. Aussi le droit à la santé fait-il partie des droits humains fondamentaux reconnus par les institutions internationales et se trouve-t-il inscrit dans la constitution de la plupart des pays. En Côte d'Ivoire, pour permettre l'accès aux services de santé, l'État ivoirien a dû opter au départ pour la gratuité des soins. Mais cette politique s'est avérée non seulement assez onéreuse pour les finances publiques mais encore porteuse d'un double déséquilibre d'une part, entre Abidjan et les villes de provinces et, d'autre part, entre les zones urbaines et les milieux ruraux (PNDS, 2016). Cette option a été progressivement abandonnée à partir de 1978. Parallèlement, la Côte d'Ivoire a souscrit à divers objectifs et principes définis à des niveaux supranationaux pour affiner et conduire sa politique sanitaire nationale. La déclaration d'Alma Ata en 1978 qui met en exergue les soins de santé primaire; l'approche District en 1985 qui privilégie le développement de secteurs sanitaires basés sur une unité opérationnelle ; l'initiative de Bamako en 1987 centrée sur l'appel à la participation communautaire pour le développement sanitaire ; et en 2000, les Objectifs du millénaire pour la santé (OMS) issus des Objectifs du millénaire pour le développement (OMD). Bien que ces différentes politiques aient permis 
d'obtenir de nombreux acquis, l'accès des populations aux services de santé demeure un problème majeur pour encore un grand nombre de population. Grand-Bassam est situé au sud-est de la Côte d'Ivoire. Dans cette ville, les populations éprouvent des difficultés pour accéder aux services de santé. Selon le RASS (2015), 25\% de la population de Grand-Bassam n'utilisent toujours pas les services de santé. Cette réalité montre qu'il existe une partie importante de la population qui n'a toujours pas accès aux services de santé. Ainsi, l'on se demande quelles sont les facteurs qui déterminent l'accès aux services de santé ? L'objectif de cet article est d'identifier les déterminants de l'accès aux services de santé dans la ville de Grand-Bassam.

\section{Outils et méthodes}

Pour atteindre l'objectif fixé, notre démarche méthodologique s'est appuyée premièrement sur une exploitation de la littérature. Les ouvrages relatifs à la santé de plusieurs bibliothèques et instituts de recherche, notamment l'Institut de Géographie Tropicale (IGT), l'Institut de Recherches et de Développement (IRD), ont été consultés ainsi que les rapports statistiques de l'Institut National de la Statistique (Recensement Général de la Population et de l'Habitat) et du ministère de la Santé (Rapport des annuaires statistiques de la santé). La seconde phase de collecte de données a porté sur l'enquête par questionnaire. A cet effet un questionnaire a été administré aux ménages de Grand-Bassam dans le mois de Mars 2017.

Le questionnaire a été administré à 576 ménages choisis à partir d'une méthode d'échantillonnage non probabiliste par choix raisonné. Il a porté sur les caractéristiques socio-économiques des chefs de ménages et la fréquentation des services de santé. Les données recueillies ont été traitées au niveau statistique avec le logiciel Microsoft Excel et SPSS. La variable dépendante (l'accès aux services de santé) a été croisée avec les variables indépendantes (sexe, âge, niveau d'instruction, taille du ménage et revenu) afin de rechercher d'éventuelles dépendances qui permettraient d'expliquer le choix pour les ménages de recourir ou non aux formations sanitaires publiques. À cet effet, le test de $\mathrm{khi}^{2}$ a été appliqué au seuil de $5 \%$.

- si la valeur de p ou Signification asymptotique est supérieure à 0,05 l'on devra accepter l'hypothèse nulle (H0) et conclure qu'il n'y a pas de lien entre l'accès aux services de santé et les différentes variables testées.

- si la valeur de p ou Signification asymptotique est inférieure à 0,05 , dans ce cas, l'hypothèse 1 (H1) est vraie ; une dépendance existe.

La figure 1 ci-dessous présente la ville de Grand-Bassam 


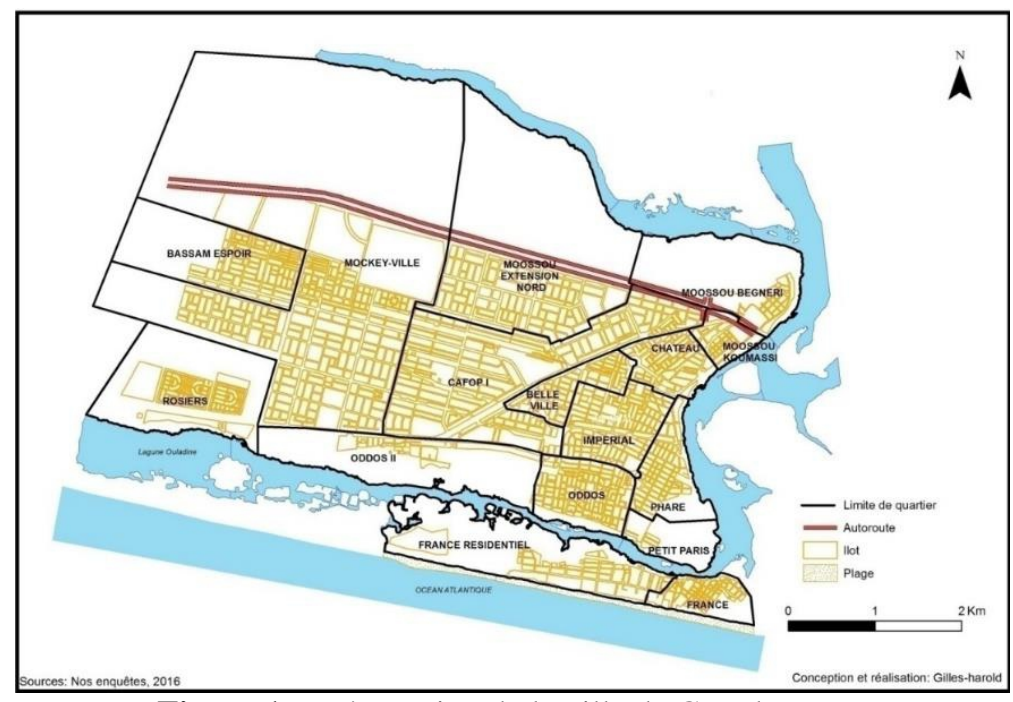

Figure 1 : Présentation de la ville de Grand-Bassam

\section{Résultats}

Caractéristiques sociodémographiques et économiques des ménages enquêtés

L'enquête a porté sur un échantillon de 576 ménages dont les caractéristiques socio démographiques et économiques sont contenues dans les tableaux ci-dessous.

- Structure par âge et par sexe des ménages enquêtés

Le tableau ci-dessous présente la répartition des ménages enquêtés selon l'âge et le sexe

Tableau 1: structure par âge et par sexe

\begin{tabular}{|c|c|c|c|}
\hline Classe d'âge & Masculin & Féminin & Total \\
\hline$[20-30[$ & 47 & 17 & 64 \\
\hline$[30-40[$ & 129 & 60 & 189 \\
\hline$[40-50[$ & 139 & 48 & 187 \\
\hline$[50-60[$ & 44 & 33 & 77 \\
\hline$[60-70[$ & 37 & 4 & 41 \\
\hline Plus de 70 & 5 & 13 & 18 \\
\hline Total & 401 & 175 & 576 \\
\hline
\end{tabular}

Source : Nos enquêtes 2017

Du tableau ci-dessus, plusieurs constats se dégagent. La structure par sexe montre que Les chefs de ménages enquêtés sont à majorité de sexe masculin. Ils sont au nombre de 475 soit une proportion de 82,46\%. Concernant les classes d'âges, on constate que les ménages dont l'âge est compris entre [30-40[ et [40-50[ sont les plus nombreux. Ils représentent 
respectivement $32,81 \%$ et $32,46 \%$ des ménages enquêtés. Les ménages ayant plus de 70 ans sont les moins nombreux. Ils représentent 3,12\% des ménages enquêtés.

\section{- Répartition des ménages selon le niveau d'étude}

Le tableau contient la répartition des chefs de ménages selon le niveau d'étude.

Tableau 2: Répartition des chefs de ménage selon le niveau d'étude

\begin{tabular}{|l|l|}
\hline Niveau d'instruction & Effectifs \\
\hline Aucun & 46 \\
\hline École coranique & 31 \\
\hline Primaire & 77 \\
\hline Secondaire & 190 \\
\hline Supérieur & 232 \\
\hline Total & 576 \\
\hline
\end{tabular}

Source : Nos enquêtes 2017

On observe à travers le tableau ci-dessus que $40,27 \%$ des ménages enquêtés ont un niveau primaire et $33 \%$ ont un niveau secondaire. Les ménages ayant un niveau primaire sont les moins nombreux. Au nombre de 31 , ils représentent $5,38 \%$ des ménages enquêtés. Par ailleurs, il existe à Grand-Bassam, des ménages sans niveau d'instruction. Ils sont au nombre de 46 soit $8 \%$ des ménages enquêtés.

\section{- Taille des ménages}

Elle se veut d'exprimer le nombre d'individus vivant sous le toit et la responsabilité des chefs de ménages.

Dans le tableau qui suit, sont enregistrées les données relatives au membre des ménages.

Tableau 3 : Taille des ménages

\begin{tabular}{|l|l|}
\hline Taille des ménages & Effectif \\
\hline$[0-4[$ & 204 \\
\hline$[4-8[$ & 253 \\
\hline$[8-12[$ & 83 \\
\hline Plus de 12 & 36 \\
\hline Total & 576 \\
\hline
\end{tabular}

Source : Nos enquêtes 2017

L'analyse du tableau montre que $44 \%$ des ménages enquêtés sont dans des ménages dont la taille est comprise dans l'intervalle [4-8 [ et 35,4\% se trouvent dans des ménages dont la taille est comprise dans l'intervalle [04[. Aussi on remarque qu'il existe moins de ménages parmi les enquêtés ayant une taille de ménages de plus de 12 personnes. Ils représentent 
- Répartition des ménages selon le revenu

Le tableau ci-dessous montre la répartition des ménages enquêtés selon le revenu

Tableau 4 : Répartition des chefs de ménage selon le revenu

\begin{tabular}{|l|l|}
\hline Revenu des ménages & Effectifs \\
\hline moins de 50000 & 51 \\
\hline $50000-100000$ & 150 \\
\hline $100000-150000$ & 92 \\
\hline $150000-200000$ & 88 \\
\hline plus de 200000 & 195 \\
\hline Total & 576 \\
\hline
\end{tabular}

Source : Nos enquêtes 2017

L'observation de ce tableau montre que $33,85 \%$ des enquêtés ont un revenu mensuel supérieur à 200.000 FCFA ; ce sont des salariés du publics majoritairement ainsi que ceux du privés. Et, $15,27 \%$ sont de la tranche [150 000-200 000[ et 15,97\% ont un revenu compris entre [100 000-150 000[ ; l'on retrouve dans ces catégories aussi, les salariés du privé et les commerçants. $26,04 \%$ des chefs de ménage ont un revenu oscillant dans l'intervalle [50 000-100 000[; ces derniers travaillent majoritairement dans le secteur informel. Enfin 8,85\% des chefs de ménage enquêtés ont déclaré avoir un revenu inférieur à $50.000 \mathrm{fcfa}$; l'on retrouve dans ces catégories, les petits commerçants et les ménagères vivant « au jour le jour ».

\section{Les déterminants de l'accès aux services de santé à Grand-Bassam}

$80,9 \%$ des ménages enquêtés ont déclaré avoir recouru à un centre de santé au cours de l'année. Les facteurs qui déterminent l'accès aux soins de santé à Grand-Bassam sont ci-dessous.

\section{- Le déterminant géographique}

Il est relatif à la perception qu'ont les chefs de ménage de la distance de leur lieu d'habitation à la structure de santé la plus proche.

Les hypothèses formulées sont les suivantes :

H0: La distance entre les structures de santé et les ménages n'a pas d'influence sur leur accès aux services de santé dans les structures sanitaires de Grand-Bassam.

H1: La distance entre les structures de santé et les ménages influence leur accès aux services de santé dans les structures sanitaires de GrandBassam.

Tableau 5 : Perception de la distance et accès aux services de santé

\begin{tabular}{|l|c|c|c|}
\hline \multirow{2}{*}{ Perception de la distance } & \multicolumn{2}{|c|}{ Accès aux services de santé } & \multirow{2}{*}{ Total } \\
\cline { 2 - 3 } & Non & Oui & \\
\hline
\end{tabular}




\begin{tabular}{|c|c|c|c|c|}
\hline & & & & \\
\hline \multirow[t]{2}{*}{ Courte } & Effectif & 28 & 167 & \multirow[t]{2}{*}{195} \\
\hline & $\begin{array}{c}\text { Effectif } \\
\text { théorique }\end{array}$ & 37,24 & 157,76 & \\
\hline \multirow[t]{2}{*}{ Acceptable } & Effectif & 15 & 114 & \multirow[t]{2}{*}{129} \\
\hline & $\begin{array}{c}\text { Effectif } \\
\text { théorique }\end{array}$ & 24,64 & 104,36 & \\
\hline \multirow[t]{2}{*}{ Longue } & Effectif & 67 & 185 & \multirow[t]{2}{*}{252} \\
\hline & $\begin{array}{c}\text { Effectif } \\
\text { théorique }\end{array}$ & 48,12 & 203,88 & \\
\hline Total & Effectif & 110 & 466 & 576 \\
\hline
\end{tabular}

Source : Nos enquêtes 2017 et les calculs du logiciel SPSS.

Test Khi-deux

\begin{tabular}{|c|c|c|}
\hline Khi-deux de Pearson & Ddl & $\begin{array}{c}\text { Signification asymptotique } \\
\text { (bilatérale) ou P }\end{array}$ \\
\hline 16.61 & 2 & 0.0002 \\
\hline
\end{tabular}

Source les calculs du logiciel SPSS, 2017.

La signification asymptotique ou $\mathrm{P}$ a une valeur de 0.0002 ce qui est inférieur à 0,05 (seuil de signification). L'hypothèse 0 dans ce cas est rejetée et l'hypothèse 1, l'hypothèse de dépendance est acceptée. Alors, la fréquentation des établissements de santé à Grand-Bassam est influencée par la perception de la distance des chefs de ménage.

Les équipements sanitaires sont inégalement répartis dans la ville (Voir figure 2). Ils sont concentrés dans les quartiers Impérial, Phare et France résidentiel. Les quartiers France,

Cafop et Moossou ne disposent qu'un seul établissement sanitaire. On ne dénombre aucune structure sanitaire dans les autres quartiers de la ville.

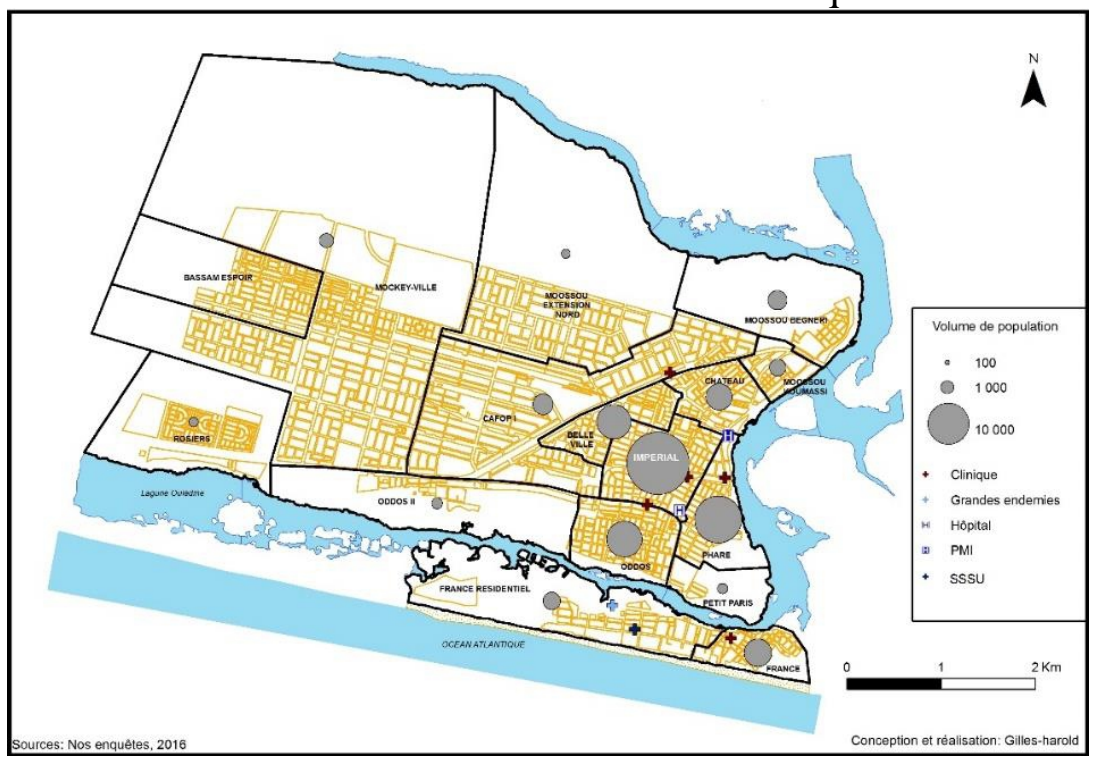

Figure 2 : Répartition de la population et des structures sanitaires à Grand-Bassam 
Selon notre enquête, $19,09 \%$ des ménages n'ont pas accès aux services de santé. Parmi les ménages n'ayant pas accès aux services de santé, on constate que $61 \%$ jugent que la distance entre leur lieu d'habitation et le centre de santé est longue. Ces ménages sont majoritairement dans mes quartiers Mockeyville et Moosou extension nord.

\section{- Les déterminants économiques}

Les variables testées sont: le revenu des chefs de ménage et la perception qu'ils ont du coût des prestations et des soins de santé.

\section{Lien entre revenu et fréquentation des hôpitaux}

Les hypothèses sont les suivantes :

H0: L'accès aux structures de santé de Grand-Bassam est indépendant du revenu des populations de ces villes.

H1: L'accès des structures de santé de Grand-Bassam est dépendant du revenu des populations de ces villes.

Tableau 6 : Revenu et accès aux services de santé

\begin{tabular}{|c|c|c|c|c|}
\hline \multirow{2}{*}{ Revenu } & \multicolumn{2}{|c|}{$\begin{array}{c}\text { Accès aux services de } \\
\text { santé }\end{array}$} & \multirow{2}{*}{ Total } \\
\cline { 2 - 4 } & Effectif & Non & Oui & \multirow{2}{*}{51} \\
\hline \multirow{2}{*}{ Moins de 50000 } & Effectif théorique & 9,74 & 41,26 & \\
\cline { 2 - 4 } & Effectif & 45 & 105 & \multirow{2}{*}{150} \\
\hline \multirow{2}{*}{$50000-100000$} & Effectif théorique & 28,65 & 121,35 & \\
\hline & Effectif & 14 & 78 & \multirow{2}{*}{92} \\
\cline { 2 - 4 } & Effectif théorique & 17,57 & 74,44 & \\
\hline 1500000-200000 & Effectif & 2 & 86 & \multirow{2}{*}{88} \\
\cline { 2 - 4 } & Effectif théorique & 16,81 & 71,19 & \\
\hline Plus de 200000 & Effectif & 10 & 185 & \multirow{2}{*}{195} \\
\cline { 2 - 4 } & Effectif théorique & 37,23 & 157,76 & \\
\hline Total & Effectif & 110 & 466 & 576 \\
\hline
\end{tabular}

Source : Nos enquêtes 2017 et les calculs du logiciel SPSS.

Test de khi2

\begin{tabular}{|c|c|c|}
\hline Khi-deux de Pearson & Ddl & Signification asymptotique (bilatérale) ou P \\
\hline 161.84 & 4 & 0.0002 \\
\hline
\end{tabular}

Source : les calculs du logiciel SPSS, 2017.

La signification asymptotique ou $\mathrm{P}$ a une valeur de 0.0002 ; ce $\mathrm{P}$ est inférieur à 0,05 (seuil de signification). L'hypothèse 0 dans ce cas est rejetée et l'hypothèse 1, l'hypothèse de dépendance est acceptée. Alors, la fréquentation des établissements de santé à Grand-Bassam dépend du revenu des chefs de ménage. 


\section{Lien entre perception du coût et accès aux services de base}

Pour établir le lien entre la perception du coût et l'accès aux services de base, nous formulons les hypothèses suivantes :

H0: L'accès aux services de santé de Grand-Bassam est indépendant de la perception du coût des prestations des soins qu'ont les chefs de ménage.

H1: L'accès aux services de santé de Grand-Bassam est dépendant de la perception du coût des prestations des soins qu'ont les chefs de ménage.

Tableau 7: Perception du coût et accès aux services de santé

\begin{tabular}{|c|c|c|c|c|}
\hline \multicolumn{2}{|c|}{ Perception des coûts des } & \multicolumn{2}{|c|}{ Accès aux soins de santé } & \multirow[t]{2}{*}{ Total } \\
\hline & & Non & Oui & \\
\hline \multirow[t]{2}{*}{ Bas } & Effectif & 20 & 190 & \multirow[t]{2}{*}{210} \\
\hline & Effectif théorique & 40,1 & 169,89 & \\
\hline \multirow{2}{*}{ Satisfaisant } & Effectif & 23 & 113 & \multirow[t]{2}{*}{136} \\
\hline & Effectif théorique & 25,97 & 110,03 & \\
\hline \multirow[t]{2}{*}{ Élevé } & Effectif & 67 & 163 & \multirow[t]{2}{*}{230} \\
\hline & Effectif théorique & 43,92 & 186,08 & \\
\hline Total & Effectif & 110 & 466 & 576 \\
\hline
\end{tabular}

Source : Nos enquêtes 2017 et les calculs du logiciel SPSS.

Test de khi 2

\begin{tabular}{|c|c|c|}
\hline Khi-deux de Pearson & Ddl & $\begin{array}{c}\text { Signification asymptotique (bilatérale) } \\
\text { ou P }\end{array}$ \\
\hline 27.87 & 2 & 0,009 \\
\hline
\end{tabular}

Source : les calculs du logiciel SPSS, 2017.

La signification asymptotique ou P a une valeur de l'ordre de 0,009 . Cette valeur est inférieure à 0,05 (seuil de signification). L'hypothèse 0 dans ce cas est rejetée et l'hypothèse 1 , l'hypothèse de dépendance est acceptée. Alors, la fréquentation des établissements de santé à Grand-Bassam est influencée par la perception qu'ont les chefs de ménage du coût des prestations sanitaires.

\section{- Les déterminants sociodémographiques}

Les variables testées sont : le sexe, le niveau d'instruction, la taille du ménage et l'âge.

\section{Lien entre le sexe et l'accès aux services de santé}

Pour établir le lien entre le sexe et l'accès aux services de santé, les hypothèses suivantes ont été formulées

H0: L'accès aux services de santé de Grand-Bassam est indépendant du sexe de la population.

H1: L'accès aux services santé de Grand-Bassam est dépendant du sexe de la population. 
Tableau 8: Sexe et accès aux services de santé

\begin{tabular}{|c|c|c|c|c|}
\hline & \multirow[b]{2}{*}{ Sexe } & \multicolumn{2}{|c|}{$\begin{array}{c}\text { Accès aux services de } \\
\text { santé }\end{array}$} & \multirow[t]{2}{*}{ Total } \\
\hline & & Non & Oui & \\
\hline \multirow[t]{2}{*}{ Féminin } & Effectif & 25 & 150 & \multirow[t]{2}{*}{175} \\
\hline & Effectif théorique & 33,42 & 141,58 & \\
\hline \multirow[t]{2}{*}{ Masculin } & Effectif & 85 & 316 & \multirow[t]{2}{*}{401} \\
\hline & Effectif théorique & 76,58 & 324,42 & \\
\hline Total & Effectif & 110 & 466 & 576 \\
\hline
\end{tabular}

Source : Nos enquêtes 2017 et les calculs du logiciel SPSS.

Tests de Khi2

\begin{tabular}{|c|c|c|}
\hline Khi-deux de Pearson & Ddl & $\begin{array}{c}\text { Signification asymptotique (bilatérale) } \\
\text { ou P }\end{array}$ \\
\hline 3.77 & 1 & 0.0521 \\
\hline
\end{tabular}

Source : les calculs du logiciel SPSS, 2017.

La signification asymptotique ou $\mathrm{P}$ a une valeur de 0.0521 qui est supérieure à 0,05 (seuil de signification). L'on peut alors rejeter l'hypothèse 1 et accepté l'hypothèse 0 . Alors, la fréquentation des établissements de santé à Grand-Bassam n'est pas influencée par le sexe des chefs de ménage.

\section{Lien entre taille du ménage et accès aux services de base}

Les hypothèses suivantes sont émises pour établir le lien entre la taille du ménage et l'accès aux services de santé.

H0: L'accès aux services de santé de Grand-Bassam est indépendant du nombre d'individus dans les ménages.

H1: L'accès aux services de santé de Grand-Bassam est dépendant du nombre d'individus dans les ménages.

Tableau 9 : taille du ménage et accès aux services de santé

\begin{tabular}{|c|c|c|c|c|}
\hline \multirow{2}{*}{\multicolumn{2}{|c|}{ Taille du ménage }} & \multicolumn{3}{|c|}{ Accès aux services de santé } \\
\hline & & Non & Oui & Total \\
\hline \multirow[b]{2}{*}{$0-4[$} & Effectif & 39 & 165 & \multirow[t]{2}{*}{204} \\
\hline & Effectif théorique & 38,96 & 165,04 & \\
\hline \multirow[b]{2}{*}{$4-8[$} & Effectif & 32 & 221 & \multirow[t]{2}{*}{253} \\
\hline & Effectif théorique & 48,32 & 204,68 & \\
\hline \multirow[b]{2}{*}{$8-12[$} & Effectif & 15 & 68 & \multirow[t]{2}{*}{83} \\
\hline & Effectif théorique & 15,85 & 67,15 & \\
\hline \multirow[b]{2}{*}{ Plus de 12} & Effectif & 24 & 12 & \multirow[t]{2}{*}{36} \\
\hline & Effectif théorique & 6,88 & 29,13 & \\
\hline Total & Effectif & 110 & 466 & 576 \\
\hline
\end{tabular}

Source : Nos enquêtes 2017 et les calculs du logiciel SPSS.

Tests de khi 2

\begin{tabular}{|c|c|c|}
\hline Khi-deux de Pearson & Ddl & Signification asymptotique (bilatérale) ou P \\
\hline 59.54 & 3 & 0 \\
\hline
\end{tabular}

Source : les calculs du logiciel SPSS, 2017. 
La signification asymptotique ou $\mathrm{P}$ est nulle. Ce chiffre est inférieur à 0,05 (seuil de signification). L'hypothèse 0 est ainsi rejetée et l'hypothèse 1 est acceptée. Alors, la fréquentation des établissements de santé à GrandBassam dépend de la taille des ménages.

\section{Lien entre niveau d'instruction et accès aux services de santé}

Pour établir le lien, nous avons formulés les hypothèses ci-dessous :

H0: L'accès aux services de santé de Grand-Bassam est indépendant du niveau d'instruction des chefs de ménages.

H1: L'accès aux services de santé de Grand-Bassam est dépendant du niveau d'instruction des chefs de ménages.

Tableau 10 : niveau d'instruction et accès aux services de santé

\begin{tabular}{|c|c|c|c|c|}
\hline \multirow{2}{*}{\multicolumn{2}{|c|}{ Niveau d'instruction }} & \multicolumn{2}{|c|}{ Accès aux services de santé } & \multirow[t]{2}{*}{ Total } \\
\hline & & \multirow{2}{*}{$\begin{array}{l}\text { Non } \\
37 \\
\end{array}$} & \multirow{2}{*}{$\begin{array}{c}\text { Oui } \\
9\end{array}$} & \\
\hline \multirow[b]{2}{*}{ Aucun } & Effectif & & & \multirow[t]{2}{*}{46} \\
\hline & Effectif théorique & 8,78 & 37,22 & \\
\hline \multirow[t]{2}{*}{ Coranique } & Effectif & 24 & 7 & \multirow[t]{2}{*}{31} \\
\hline & Effectif théorique & 5,92 & 25,08 & \\
\hline \multirow{2}{*}{ Primaire } & Effectif & 40 & 37 & \multirow[t]{2}{*}{77} \\
\hline & Effectif théorique & 14,7 & 62,3 & \\
\hline \multirow[t]{2}{*}{ Secondaire } & Effectif & 6 & 184 & \multirow[t]{2}{*}{190} \\
\hline & Effectif théorique & 36,28 & 153,72 & \\
\hline \multirow[t]{2}{*}{ Supérieur } & Effectif & 3 & 229 & \multirow[t]{2}{*}{232} \\
\hline & Effectif théorique & 44,31 & 187,69 & \\
\hline Total & Effectif & 110 & 466 & 576 \\
\hline
\end{tabular}

Source : Nos enquêtes 2017 et les calculs du logiciel SPSS.

Tests de Khi 2

\begin{tabular}{|c|c|c|}
\hline Khi-deux de Pearson & Ddl & Signification asymptotique (bilatérale) ou P \\
\hline 312.99 & 4 & 0 \\
\hline
\end{tabular}

Source : les calculs du logiciel SPSS, 2017.

La signification asymptotique ou $\mathrm{P}$ est nulle. L'hypothèse nulle (ou l'hypothèse d'indépendance) dans ce cas est rejetée, l'hypothèse 1 (ou de dépendance) est acceptée. Alors, la fréquentation des établissements de santé à Grand-Bassam est influencée par le niveau d'instruction des chefs de ménage.

\section{Lien entre âge et accès aux services de santé}

Pour établir le lien entre l'âge et l'accès aux services de santé, nous avons formulées les hypothèses suivantes :

H0: L'accès aux services de santé de Grand-Bassam est indépendant de l'âge des chefs de ménages. 
H1: L'accès aux services de santé de Grand-Bassam est dépendant de l'âge des chefs de ménages.

Tableau 11: âge et accès aux services de santé

\begin{tabular}{|c|c|c|c|c|}
\hline & \multirow[t]{2}{*}{ Âge } & \multicolumn{2}{|c|}{ Accès aux soins de santé } & \multirow[b]{2}{*}{ Total } \\
\hline & & Non & Oui & \\
\hline \multirow[b]{2}{*}{$20-30[$} & Effectif & 20 & 44 & \multirow[b]{2}{*}{64} \\
\hline & Effectif théorique & 12,22 & 51,78 & \\
\hline \multirow[b]{2}{*}{$30-40[$} & Effectif & 34 & 165 & \multirow[b]{2}{*}{189} \\
\hline & Effectif théorique & 36,1 & 152,9 & \\
\hline \multirow[b]{2}{*}{$40-50[$} & Effectif & 36 & 141 & \multirow[b]{2}{*}{177} \\
\hline & Effectif théorique & 35,12 & 151,28 & \\
\hline \multirow[b]{2}{*}{ [50-60[ } & Effectif & 17 & 60 & \multirow[b]{2}{*}{77} \\
\hline & Effectif théorique & 14,7 & 62,3 & \\
\hline \multirow[b]{2}{*}{ [60 et plus } & Effectif & 3 & 38 & \multirow[b]{2}{*}{41} \\
\hline & Effectif théorique & 7,82 & 33,17 & \\
\hline \multirow[b]{2}{*}{ Plus de 70} & Effectif & 0 & 18 & \multirow[b]{2}{*}{18} \\
\hline & Effectif théorique & 3,43 & 14,57 & \\
\hline Total & Effectif & 110 & 466 & 576 \\
\hline
\end{tabular}

Source : Nos enquêtes 2017 et les calculs du logiciel SPSS.

Test du khi-deux

\begin{tabular}{|c|c|c|}
\hline Khi-deux de Pearson & Ddl & $\begin{array}{c}\text { Signification asymptotique (bilatérale) } \\
\text { ou P }\end{array}$ \\
\hline 15,18 & 5 & 0.0096 \\
\hline
\end{tabular}

Source : les calculs du logiciel SPSS, 2017.

La signification asymptotique ou P est de l'ordre de 0.0096 ; ce qui est inférieur à 0,05 (seuil de signification). L'hypothèse 0 est ainsi infirmée et l'hypothèse 1 est acceptée. Alors la fréquentation des établissements de santé à Grand-Bassam dépend de l'âge des ménages.

\section{Discussion}

Les résultats de cette étude montrent que la perception de la distance, le revenu du chef de ménage, la perception du coût des soins, la taille des ménages, le niveau d'instruction et l'âge des ménages sont les facteurs qui déterminent l'accès des populations aux services de santé dans la ville de Grand-Bassam.

La perception de la distance des chefs de ménages à Grand-Bassam est déterminante dans leur accès aux services de santé. Ces résultats sont partagés par Houffouet (2015) qui montre que la perception de la distance a une influence sur l'accès des populations aux services de santé dans la ville d'Agboville. Elle nous apprend que 86,85\% des ménages à Agboville disant ne pas recourir aux centres de soins en cas de maladie, ont une perception 
différente de la distance: elle est longue. Certes, il n'existe pas de problème d'accès physique aux soins de santé d'autant plus qu'ils sont tous situés en bordure de route à l'exception de ' $^{\prime} \mathrm{AGEM}^{1}$ et que la voirie de la ville est globalement en bon état. Mais l'inégale répartition de ces dernières fait que la distance à parcourir pour les populations des quartiers Nord, Nord-est, Nord-ouest semble plus longue et plus coûteuse. Dans la même veine, Doumbouya (2008) affirme que dans les zones rurales de la Guinée, la perception de la distance d'accès aux centres de santé était un facteur déterminant de l'accès car95\% des réponses des usagers ruraux enquêtés le dénoncent comme facteur d'iniquité d'accès. Cependant pour Améa (2015), la distance géographique ne constitue pas un obstacle majeur à l'accès aux soins sanitaires à Bonoua même si l'organisation spatiale a révélé une inégalité dans la répartition spatiale des structures sanitaires. Comme Améa, N'Doli (2016) a montré que la perception de la distance ne constitue pas un obstacle dans l'accès aux services de santé dans la ville de Dabou.

Le revenu du chef de ménage influence également l'accès aux services de santé dans la ville de Grand-Bassam. Haddad et al.(2016) font le même constat lorsqu'ils affirment que l'accès aux services de santé au Burkina Faso est déterminé par plusieurs facteurs. La fréquentation des centres de santé et la consommation de soins sont fortement contraintes par la capacité de paiement des ménages. $70 \%$ des personnes ayant été malades dans les quatre semaines précédant l'entrevue pensent qu'elles auraient pu mieux se soigner si elles avaient eu plus d'argent. Les ménages ayant rapporté plus de difficultés à faire face aux besoins de santés ont les plus pauvres, les moins éduqués, ceux vivant en milieu rural et loin d'un centre de santé. Tapé (2014) abonde dans le même sens. Selon lui, le revenu des ménages du quartier Sicogi ${ }^{2}$ a une influence sur leur choix de recourir aux établissements de soins en cas de besoins.

Notre étude a montré que la perception qu'ont les chefs de ménages sur les coûts des soins influence leurs recours aux centres de santé à GrandBassam. Ce résultat est conforme à celui obtenu par Houffouet (op.cit.) qui nous apprend que la perception que la population d'Agboville se fait des coûts des prestations et des soins pratiqués dans les structures sanitaires a une influence sur son choix de recours aux soins en cas de besoin. Tapé (op.cit.) fait le même constat dans son étude à Yopougon-sicogi. Il affirme que la perception qu'a la population des coûts de prestation et des soins pratiqués dans les structures sanitaires de Yopougon-Sicogi a une influence sur leur choix de recours aux soins en cas de besoin.

${ }^{1}$ AGEM : Agnéby Espace Médical, est un établissement sanitaire de la ville d'Agboville.

${ }^{2}$ Sicogi : quartier de la commune de Yopougon. 
Le niveau d'instruction des chefs de ménages influence également l'accès aux services de santé à Grand-Bassam. Ce résultat confirme celui de Houffouet (op.cit.) qui affirme que le niveau d'instruction des chefs de ménages à Agboville influence leur recours aux soins dans les structures sanitaire. Améa (op.cit.) partage ce résultat. Selon elle, plus le chef de ménage à un niveau d'instruction élevé, plus celui-ci recourt aux centres de santé. En revanche moins le chef de ménage est instruit moins il recourt aux centres de santé. Dans le même sens Tapé (op.cit.) affirme que le niveau d'étude des chefs de ménages à Yopougon-sicogi influence leur recours aux soins dans les structures sanitaires.

L'impact de l'âge dans la fréquentation des centres de santé a été souligné par Houffouet (op.cit.). Ce résultat est similaire au notre. Selon elle, les jeunes chefs de ménage sollicitent plus les établissements de santé que les vieux dans la ville d'Agboville. Ce résultat est conforté par celui de Tapé (op.cit.) qui affirme que l'âge influence le recours aux soins de santé dans les structures sanitaires de Yopougon-Sicogi.

L'accès aux services de santé à Grand-Bassam est aussi influencé par la taille du ménage. Ce résultat est conforme à celui de Loba et al. (2015) dans leur travail sur les déterminants du recours aux formations sanitaires publiques à Abidjan. Selon eux, plus le ménage est grand, moins il y a de chance au vu du seuil du revenu de 30000 F CFA de solliciter la formation publique. Les populations les plus économiquement vulnérables courent ainsi le risque d'une marginalisation sanitaire accrue. Pour Haddad et al.(op.cit.), les personnes vivant dans les ménages comptant cinq personnes et plus ont une probabilité plus élevée de rencontrer des difficultés à faire face à leurs besoins de santé que ceux des plus petits ménages (d'une à quatre personnes). La survenue de ces difficultés est deux fois plus fréquente dans les ménages de dix personnes et plus. Améa (op.cit.) abonde dans le même sens car selon elle, la taille du ménage conditionne l'accès aux soins dans les centres de santé de Bonoua. Selon elle, plus le ménage est de grande taille moins celui-ci recourt aux centres de santé. A l'inverse, plus la taille du ménage est petite, plus le taux de recours du ménage aux centres de santé est élevé.

Notre étude montre également que le sexe n'a aucune influence sur l'accès aux services de santé. Ce résultat est conforme est celui trouvé par Mariko et al. (2000) qui n'ont trouvé aucun lien entre le sexe et l'accès aux soins. Ils ont montré que dans les villes de Niamey et Dakar, le sexe n'est pas un facteur d'utilisation quel que soit le type de formation sanitaire. Le résultat trouvé par Ymba (2013) est contraire au notre. Selon elle, du fait de leur morphologie biologique les femmes expriment généralement une plus grande demande de soins de santé (soins spécialisés tels que les soins gynécologiques, les moyens de contraception, etc.) que les hommes. Le sexe 
influence donc l'accès aux services de santé. Costas et al. (2003), ont un résultat similaire à celui de Ymba. Leur une étude sur les difficultés d'accès aux services de premier contact au canada a révélé que le sexe influence leur accès aux services de santé.

\section{Conclusion}

Au terme de cette étude, il ressort que dans la ville de Grand-Bassam, l'accès aux services de santé est déterminé par des facteurs sociodémographiques, économiques et démographiques. En effet, par le biais du test de $\mathrm{Khi}^{2}$, les déterminants recensés sont : la perception de la distance, le revenu du chef de ménage, la perception du coût, la taille du ménage, le niveau d'instruction et l'âge des chefs de ménage. Il appartiendra aux autorités en charge de l'espace urbain de mettre en place des stratégies en vue de rapprocher les populations des centres de santé tant sur le plan de la distance géographique que des ressources économiques

\section{References:}

1. AMEA (2015), Structures sanitaires et accès aux soins de santé dans la ville de Bonoua, mémoire de master en géographie de la santé, UFHB. 180 p.

2. COSTAS K., RAYNALD P. (2003), besoins et difficultés d'accès aux services de premier contact, Canada, Québec, Montréal. Direction de santé publique de l'Agence de la santé et des services sociauxde Montréal, 102P.

3. DOUMBOUYA M.L. (2008), Accessibilité des services de santé en Afrique de l'Ouest : le cas de la Guinée. In :Workingpaper $n^{\circ}$ 2008-2, Lyon : Université de Lyon II, $20 \mathrm{p}$

4. ÉRIC F., RODRIGUE L. (2014), L'accès aux services de santé en français et leur utilisation en contexte francophone minoritaire. Société Santé en Français, 158 P.

5. HADDAD S., NOUGTARA A., RIDDE V. (2004) Les inégalités d'accès aux services de santé et leurs déterminants auBurkina Faso. In: Santé, Société et Solidarité, $n^{\circ} 2$. Inégalités sociales de santé. pp. 199-210;

6. HOUFFOUET J. (2015), infrastructures sanitaires et accès aux soins de santé à Agboville, mémoire de master en géographie de la santé, UFHB. 159 p.

7. JEANINE C. (2016), Difficulté liée à l'accèsaux services de soins de santé au Canada. In Coup d'oil sur la santé, No 82, 624-X, P 1-11

8. LOBA A. V., BOSSON E (2015), Les déterminants du recours aux formations sanitaires publiques à Abidjan (Côte d'Ivoire), in Revue 
de géographie du laboratoire Leïdi - ISSN 0851 - $2515-\mathrm{N}^{\circ} 13$, décembre 2015, P 155-170.

9. MARIE D., CLAIRE-JEHANNE D., JOSÉE D.(2014), L'accès aux services sociaux et de santé en français et laformation des professionnelles et des professionnels ensituation francophone minoritaire canadienne." In Reflets 202 (2014), P 10-19.

10. MARIKO M. et CISSE B. (2000), Équité dans l'accès aux soins et l'utilisation des services de santé : Cas de cinq capitales ouestafricains, (Rapport final). IRD, UNICEF, Coopération française, p129-130. $169 \mathrm{p}$

11. MUNYAMAHORO M, NTAGANIRA J. (2012), déterminants de l'utilisation des services de santé par les ménages du district de Rubavu, in Revue Médicale Rwandaise, P 24-31.

12. N'DOLI S. (2016), Infrastructures sanitaires et accès aux soins de santé dans la commune de Dabou, thèse de doctorat en géographie de la santé, UFHB, $284 \mathrm{p}$.

13. TAPE Bi A. (2014), L'accès et le recours aux soins de santé dans les structures sanitaires de Yopougon-Sicogi. Mémoire de master 2, IGT, UFHB. 180 p.

14. YMBA M.(2013),Accès et recours aux soins de santé modernes en milieu urbain: cas de la ville d'Abidjan, thèse de Doctorat en Géographie de la santé, Université d'Artois - Arras, 534 p. 